\title{
Inertial Taylor Columns and Jupiter's Great Red Spot ${ }^{1}$
}

\author{
ANDREW P. INGERSOLL \\ Division of Geological Sciences, California Institute of Technology, Pasadena
}

(Manuscript received 18 July 1968, in revised form 4 February 1969)

\begin{abstract}
A homogeneous fluid is bounded above and below by horizontal plane surfaces in rapid rotation about a vertical axis. An obstacle is attached to one of the surfaces, and at large distances from the obstacle the relative velocity is steady and horizontal. Solutions are obtained as power series expansions in the Rossby number, uniformly valid as the Taylor number approaches infinity.

If the height of the obstacle is greater than the Rossby number times the depth, a stagnant region (Taylor column) forms over the obstacle. Outside this region there is a net circulation in a direction opposite the rotation. The shape of the stagnant region and the circulation are uniquely determined as part of the solution.

Possible geophysical applications are discussed, and it is shown that stratification renders Taylor columns unlikely on earth, but that the Great Red Spot of Jupiter may be an example of this phenomenon, as Hide has suggested.
\end{abstract}

\section{Introduction}

Taylor (1917) and Proudman (1916) first showed that steady, weak currents in a rotating homogeneous fluid are two-dimensional: the fluid moves about in columns whose axes are parallel to the rotation vector. Moreover, since the currents tend to be deflected around solid obstacles, those columns of fluid which intersect an obstacle are isolated from the rest of the flow. These isolated regions are called Taylor columns. They were first observed in the laboratory by Taylor (1923) and more recently by Hide and Ibbetson (1966) and Hide et al. (1968). They are not observed in the earth's atmosphere, but Hide $(1961,1963)$ has suggested that the Great Red Spot is a manifestation of a Taylor column in the atmosphere of Jupiter.

However, there is no entirely satisfactory theory of Taylor columns to compare with observation, either in the laboratory or in planetary atmospheres. If an inviscid theoretical model is used, the flow depends on the initial conditions for all time (e.g., Stewartson, 1953). A steady, inviscid solution is obtained if one assumes that the fluid is initially in a state of uniform rotation, but this solution is not unique. In addition, the laboratory experiments show that the fluid within the Taylor column in stagnant, but the inviscid models with the most obvious initial conditions do not give this result.

Jacobs (1964) obtained a unique solution, valid only for slow, viscous flow in which accelerations relative to the rotating reference frame are negligible. In his model, a homogeneous fluid is contained between planes perpendicular to the rotation vector, with an axially

1 Contribution No. 1550 of the Division of Geological Sciences, California Institute of Technology, Pasadena. symmetric obstacle attached to one plane. The fluid within the Taylor column is found to be stagnant, and the streamlines are symmetric to the left and right of the obstacle, as well as upstream and downstream from it. Jacobs' solution emphasizes the importance of dissipation in determining unique steady solutions, but the relative accelerations are not negligible for most laboratory and geophysical flows. In fact, the streamlines observed by Hide and Ibbetson are decidedly asymmetric, as are the markings around Jupiter's Great Red Spot.

In this paper, we consider steady solutions which are valid when the relative accelerations predominate over the viscous accelerations. The model resembles the earlier inviscid models, but the lack of uniqueness has been removed by formulating the problem with viscosity included, and then letting the viscosity tend to zero.

The general nonlinear problem is discussed in the next section for a homogeneous fluid bounded by horizontal planes which are perpendicular to the rotation vector. The fluid is in rapid rotation, so the flow is quasi-horizontal and quasi-two-dimensional. The equation is derived for the lowest order interior stream function, which describes the flow outside the Ekman boundary layers in the limit as the Rossby number approaches zero and the Taylor number approaches infinity. Anticipating that Taylor columns will appear as regions of closed streamlines above the obstacle, we show that the fluid must be motionless within any such region in the steady state. This follows since the Ekman layer transport is always to the left of the interior velocity at both the top and the bottom surfaces. The assumption is made that the edge of the Taylor column is not a high vorticity layer. The assumption is based on 
a heuristic argument, since the appropriate nonlinear boundary layer equation has not been solved. From this condition, and the condition of no motion within the Taylor column, we derive an important free surface boundary condition which is applied on the closed portion of the critical streamline.

No attempt is made to solve the general problem, when the effects of inertia and friction are of equal importance. Solutions are obtained only in the inviscid limit, although the effects of friction are retained in the free surface boundary condition. However, the inviscid problem is still nonlinear, even when the solutions are expanded as power series in the Rossby number. This is because the location of the free surface at the edge of the Taylor column is not known a priori. The differential equation becomes linear when the flow is uniform at infinity away from the obstacle, but the nonlinearity due to the free surface boundary condition remains. Therefore, the inviscid problem with uniform flow at infinity is solved only in two special cases, but these provide insight into the solutions for more general obstacle sizes and shapes.

This paper concludes with a brief discussion of the effects of stratification, baroclinicity and the $\beta$-effect as they apply to the formation of Taylor columns in planetary atmospheres. Stratification and baroclinicity are important in the earth's atmosphere, which explains why Taylor columns are not observed on earth. However, the importance of these effects varies inversely as the horizontal scale of the phenomenon, so they may not be important in the dynamics of Jupiter's Great Red Spot. The $\beta$-effect probably is important in the flow around the Great Red Spot, but this fact is not inconsistent with Hide's hypothesis that the Spot is a Taylor column.

\section{General formulation}

Consider a homogeneous, incompressible fluid contained between two horizontal planes. The system rotates counterclockwise about a vertical axis, and velocity is measured relative to the state of uniform rotation. The obstacle is a slight irregularity in one of the boundaries, and at large distances from the obstacle the flow is uniform and horizontal. All components of the velocity must vanish at solid boundaries, and the flow is steady in the rotating reference frame.

Let $z$ be a dimensionless vertical coordinate, scaled by the distance $H$ between the planes. Let $x$ and $y$ be horizontal coordinates, also scaled by $H$. Let $u, v, w$ be the $x, y, z$ components of velocity, scaled by $V$, a characteristic velocity. Then $\mathbf{q}=(u, v, w)$ is the dimensionless velocity vector. The dimensionless quantity $p$ is the actual pressure minus the hydrostatic pressure (due to the gravitational and centrifugal accelerations), scaled by the quantity $2 \Omega V H \rho$, where $\Omega$ is the rotation rate and $\rho$ the density. Let $z=1$ be the equation of the upper boundary, and $z=h(x, y)$ that of the lower bound- ary. We assume that $h \rightarrow 0$ as $x^{2}+y^{2} \rightarrow \infty$, so $h$ is the height of the obstacle relative to the depth $H$. Then

$$
\epsilon=V /(2 \Omega H),
$$

is the Rossby number and

$$
\mathrm{R}=\Omega H^{2} / \nu,
$$

is the Taylor number, where $\nu$ is the kinematic viscosity of the fluid. In this section we consider the limit

$$
\epsilon \rightarrow 0, \quad \epsilon \mathrm{R}^{\frac{1}{2}}=\mathrm{O}(\mathbf{1}), \quad h / \epsilon=\mathrm{O}(1),
$$

corresponding to weak flow in a rapidly rotating system. The quantity $\epsilon R^{\frac{1}{2}}$ is the ratio of the time constant for decay of vorticity by Ekman layer suction [the spin-up time (Greenspan and Howard, 1963)] to the advection time $H / V$. And the quantity $h / \epsilon$ is the ratio of the topographically-induced vorticity to the vorticity $V / H$.

In this notation, the continuity equation and NavierStokes equations are

$$
\begin{gathered}
u_{x}+v_{y}+w_{z}=0, \\
\epsilon \mathbf{q} \cdot \nabla u-v=-p_{x}+\frac{1}{2 \mathrm{R}} \nabla^{2} u, \\
\epsilon \mathbf{q} \cdot \nabla v+u=-p_{y}+\frac{1}{2 \mathrm{R}} \nabla^{2} v, \\
\epsilon \mathbf{q} \cdot \nabla w=-p_{z}+\frac{1}{2 \mathrm{R}} \nabla^{2} w .
\end{gathered}
$$

From these we derive the $z$ component of the vorticity equation:

$$
\epsilon \mathbf{q} \cdot \nabla \zeta-w_{z}(1+\epsilon \zeta)+\epsilon\left(w_{x} v_{z}-w_{y} u_{z}\right)=\frac{1}{2 \mathrm{R}} \nabla^{2} \zeta,
$$

where $\zeta=v_{x}-u_{y}$ is the $z$ component of vorticity. Finally, we derive the Bernoulli equation

$$
q \cdot \nabla(p+\epsilon E)=\frac{1}{2 \mathrm{R}}\left(u \nabla^{2} u+v \nabla^{2} v+w \nabla^{2} w\right)
$$

where $E=\frac{1}{2}\left(u^{2}+v^{2}+w^{2}\right)$ is the dimensionless kinetic energy of the fluid.

In accordance with (3), we introduce an ordinary power series expansion in $\epsilon$, i.e.,

$$
u=u^{(0)}+\epsilon u^{(1)}+\epsilon^{2} u^{(2)}+\ldots
$$

It is consistent to assume that $u^{(n)}, v^{(n)}$ and $w^{(n)}$ are quantities of order unity whose derivatives with respect to $z$ are large only in the vicinity of the top and bottom surfaces. Henceforth, we shall refer only to the interior flow; modifications due to the Ekman boundary layers will be introduced as boundary conditions to be applied at $z=1$ and $z=h$. From (5) we have, for the interior flow

$$
v^{(0)}=p_{x}{ }^{(0)}, \quad u^{(0)}=-p_{y}{ }^{(0)}, \quad 0=p_{z}{ }^{(0)} ;
$$


whence from (4)

$$
u_{z}{ }^{(0)}=v_{z}{ }^{(0)}=w_{z}{ }^{(0)}=0 .
$$

Thus, to lowest order the interior flow is two-dimensional with $p^{(0)}$ as stream function.

Most theories of Ekman boundary layers are derived under the assumption $\epsilon \mathrm{R}^{\frac{1}{2}} \ll 1$ (e.g., Jacobs, 1964). When $\epsilon \mathrm{R}^{\frac{1}{2}}$ is not small, care must be exercised to ensure that the expansion in $\epsilon$ is uniformly valid over the entire range of the boundary layer coordinate. Nevertheless, in all cases which have been investigated, the condition on the interior velocity, to lower order in $\epsilon$, is the same as in the strictly linear theories (e.g., Benney, 1965; Greenspan and Weinbaum, 1965). Thus, from Jacobs' analysis, the vertical velocities at the interior edges of the Ekman boundary layers at $z=1$ and $z=h$ are

$$
-\mathrm{R}^{-\frac{1}{2} \zeta^{(0)} / 2} \text { and } u^{(0)} h_{x}+v^{(0)} h_{y}+\mathrm{R}^{-\frac{1}{2} \zeta^{(0)}} / 2 \text {, }
$$

respectively, where we have used the fact that $h$ and all its derivatives are $O(\epsilon)$. To lowest order in $\epsilon$, these boundary conditions may be applied at $z=1$ and $z=0$. From (3), these vertical velocities are $O(\epsilon)$ at most, so from (10) we conclude that $w^{(0)}=0$ in the interior of the fluid.

The differential equation for the interior stream function $p^{(0)}$ is derived from (6). To lowest order in $\epsilon$ we have $w_{z}{ }^{(0)}=0$, which is satisfied automatically by $(10)$. To first order in $\epsilon$, Eq. (6) becomes

$$
\mathbf{q}^{(0)} \cdot \nabla \zeta^{(1)}-\frac{1}{2 \epsilon \mathrm{R}} \nabla^{2} \zeta^{(0)}=w_{\mathbf{g}^{(1)}}^{(1)}
$$

where $\mathbf{q}^{(0)}$ and $\zeta^{(0)}$ may be expressed in terms of the stream function $p^{(0)}$ by (9). The left side of (12) is independent of $z$, so $w^{(1)}$ must be linear in $z$. Then with (11) we obtain

$$
\mathbf{q}^{(0)} \cdot \nabla\left[\zeta^{(0)}+\frac{h}{\epsilon}\right]=-\frac{1}{\epsilon \mathrm{R}^{\frac{1}{2}}} \zeta^{(0)}+\frac{1}{2 \epsilon \mathrm{R}} \nabla^{2} \zeta^{(0)}
$$

Eq. (13) is the required nonlinear equation for the interior stream function $p^{(0)}(x, y)$. The first term on the right side represents the rate of destruction of relative vorticity by Ekman layer suction. The second term represents the laterial diffusion of vorticity. The second term will be comparable to the first in vertical shear layers of thickness $\mathrm{R}^{-\frac{1}{\mathrm{t}}}$. Outside of vertical shear layers, the second term will be negligible.

If the flow is uniform far from the obstacle, there are streamlines $p^{(0)}(x, y)$ which terminate at infinity. Let us assume that one of these intersects itself, enclosing a region of closed streamlines. We now show that the fluid within such a region is stagnant. Integrate (13) with respect to $x$ and $y$ over the region bounded by a closed streamline. The left side is the divergence of a vector $\left[\zeta^{(0)}+h / \epsilon\right] \boldsymbol{q}^{(0)}$, which vanishes on integration because the component of $\mathbf{q}^{(0)}$ normal to the closed streamline is zero. Let us tentatively assume that there are no high-vorticity layers outside the Ekman boundary layers. Then the second term on the right will be $O(1)$ only in layers of vorticity discontinuity of thickness $\mathrm{R}^{-\frac{1}{4}}$, so the integral of the second term will be $O\left(R^{-\frac{1}{4}}\right)$. The integral of the first term on the right can be expressed as the circulation round the closed streamline divided by $\in R^{\frac{1}{2}}$. Thus, (13) implies

$$
\oint\left[\mathbf{q}^{(0)} \cdot \mathbf{d r}\right]=\mathrm{O}\left(\mathrm{R}^{-1}\right)
$$

around any closed streamline, provided the streamline does not pass through a high-vorticity region. Since the velocity $\mathbf{q}^{(0)}$ cannot change sign except on the critical streamline, where there are one or more stagnation points, (14) implies that the velocity within the region enclosed by the critical streamline must be $\mathrm{O}\left(\mathrm{R}^{-\frac{1}{4}}\right)$.

The above conclusion rests on the assumption that the vorticity $\zeta^{(0)}$ is $O(1)$. We might hope to analyze (13) using boundary layer methods in order to decide whether free shear layers can exist, but the boundary layer equation is nonlinear, and solutions have not been obtained. Instead, we present a heuristic argument to show that vorticity must be $\mathrm{O}(1)$ everywhere in the fluid. Vorticity is produced at the rate $-\mathbf{q}^{(0)} \cdot \nabla h / \epsilon$, which is $O(1)$, by (3). Ekman layer suction results simply in the destruction of vorticity, by (13). This leaves lateral diffusion, important only in vertical shear layers. Let us assume that such a shear layer exists, outside of which vorticity is $\mathrm{O}(1)$. Then on the streamline where vorticity is at its maximum, (13) implies that the downstream derivative of $\left|\zeta^{(0)}\right|$ is negative. Thus, the source of high vorticity within the shear layer must lie upstream, which is impossible since streamlines are either closed, or terminate at infinity, where the flow is uniform.

Vorticity $\zeta^{(0)}$ must be $O(1)$, although there may be layers across which it changes abruptly. If the layer thickness is $O\left(R^{-\frac{1}{2}}\right)$, as implied by (13), only the $O\left(R^{-\frac{1}{4}}\right)$ component of velocity may exhibit boundary layer structure; the $\Theta(1)$ component of velocity is continuous. Thus, we may assume that the $O(1)$ velocity can be obtained from continuous solutions of (13) without the second term on the right. As part of this assumption, the free surface boundary condition is

$$
\mathbf{q}^{(0)}=\mathbf{k} \times \nabla p^{(0)}=0, \quad\left[p^{(0)}=p_{c}^{(0)}\right],
$$

where $\mathbf{k}$ is the vertical unit vector. This condition is applied on the closed portion of the critical streamline $p^{(0)}=p_{c}{ }^{(0)}$, and serves to fix its location.

\section{The inviscid limit}

In describing the flow outside the critical streamline, we may neglect both terms on the right in (13) provided

$$
\epsilon \rightarrow 0, \quad \epsilon \mathrm{R}^{\frac{1}{2}} \rightarrow \infty, \quad h / \epsilon=\mathrm{O}(1) \text {. }
$$


The free surface boundary condition (15) was derived from (13) under the assumption that the Ekman layer suction term was $\mathrm{O}(1)$, and therefore much larger than the neglected terms of order $\epsilon, \epsilon^{2}$, etc. However, we shall show that the neglected terms do not change the free surface boundary condition (15), even when they are larger than the Ekman layer suction term. Thus, in the inviscid limit, the differential equation is (13) with right side equal to zero, and the free surface boundary condition is (15), valid uniformly as $\epsilon \rightarrow 0$ and $\epsilon \mathrm{R}^{\frac{1}{2}} \rightarrow \infty$.

The condition (15) arises because the existence of closed curves on which $p^{(0)}$ is constant implies a contradiction unless the circulation round the curve is zero. Circulation implies a net vorticity within the region, which implies a net influx of fluid from the Ekman layers, by (11). For $\epsilon \mathrm{R}^{\frac{1}{2}}=\mathrm{O}(1)$, the contradiction arises because the lateral boundary of the region is a streamline, and so there cannot be an efflux of fluid to balance the Ekman layer influx. In generalizing (15) to the case $\epsilon \mathrm{R}^{\frac{1}{2}} \rightarrow \infty$, we must show to all orders in $\epsilon$ that there cannot be an efflux from a region within a curve on which $p^{(0)}$ is constant. To do this, we consider the circulation theorem. From (5), it follows that

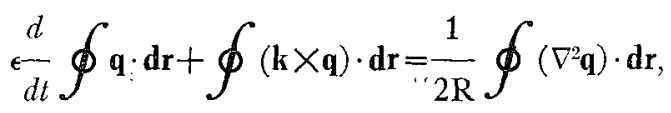

where the integral is taken around a closed contour which moves with the fluid. Moreover, if the flow is steady, and if there are closed particle paths, then the first term on the left is zero when the integral is taken around such a path.

Assume that there are closed curves in the $x-y$ plane on which $p^{(0)}$ is constant. Let $S_{0}$ be the cylindrical surface formed from one of these curves with generators parallel to the $z$ axis. Let us assume that we have proved the theorem through order $n$; in other words, that the net flux through $S_{0}$ is $\mathrm{O}\left(\epsilon^{n+1}\right)$. Then there exists a surface $S_{n}$ on which the velocity

$$
\mathbf{q}^{(0)}+\epsilon \mathbf{q}^{(1)}+\cdots+\epsilon^{n} \mathbf{q}^{(n)}
$$

is everywhere tangent to the surface. Moreover, $S_{n}$ may be made almost to coincide with $S_{0}$, the error in position being $\mathrm{O}(\epsilon)$. For the moment we neglect the influx of fluid at the top and bottom due to Ekman layer suction, as well as the term on the right in (17). Then particle paths will be nearly closed contours lying almost entirely in $S_{n}$, the error being $\mathrm{O}\left(\epsilon^{n+1}\right)$. The first term on the left in (17) will be $\mathrm{O}\left(\epsilon^{n+2}\right)$, if the integral is taken around a particle path in $S_{n}$. Therefore the second term,

$$
\begin{aligned}
\oint\left\{\mathbf{k} \times\left[\mathbf{q}^{(0)}+\epsilon \mathbf{q}^{(1)}+\cdots\right.\right. & \left.\left.+\epsilon^{n} \mathbf{q}^{(n)}\right]\right\} \cdot \mathbf{d r} \mathbf{r} \\
& +\oint\left[\mathbf{k} \times \epsilon^{n+1} \mathbf{q}^{(n+1)}\right] \cdot \mathbf{d r},
\end{aligned}
$$

taken around the same path, must alsn he $O\left(\epsilon^{n+2}\right)$. How- ever, the path element $\mathbf{d r}$ is parallel to the $n$th order approximation to the velocity (18), so the first term in (19) is zero. This leaves

$$
\oint\left[\mathbf{k} \times \mathbf{q}^{(n+1)}\right] \cdot \mathbf{d r}=\mathrm{O}(\epsilon)
$$

where the integral is taken around a particle path in $S_{n}$. This path coincides, to $\mathrm{O}(\epsilon)$, with the lowest order particle paths in $S_{0}$, so (20) is valid on the surface $S_{0}$ as well. However, the vector normal to $S_{0}$ is horizontal, so (20) is equivalent to the statement

$$
\iint \mathbf{q}^{(n+1)} \cdot \mathbf{d} \mathrm{A}_{0}=\mathrm{O}(\boldsymbol{\epsilon})
$$

where $\mathbf{d A}_{0}$ is an element of area on $S_{0}$. From (21) it follows that the net efflux throuth $S_{0}$ is $O\left(\epsilon^{n+2}\right)$, and so we have proved the theorem to one higher power of $\epsilon$.

In the preceding discussion the effects of Ekman layer suction and lateral diffusion were neglected. Now assume that $\mathrm{R}^{-\frac{1}{2}}=\mathrm{O}\left(\epsilon^{n+1}\right)$, for some non-negative integer $n$. To lowest order in $\epsilon$, the influx of fluid from the Ekman layers is still given by (11), and is therefore $O\left(\epsilon^{n+1}\right)$. Therefore, particle paths will still be closed on the surface $S_{n}$, so the proof still holds through order $n+1$, provided the term on the right in (17) is negligible. In this case the efflux through $S_{0}$ is $\mathrm{O}\left(\epsilon^{n+2}\right)$, which implies that the Ekman layer influx must also be $O\left(\epsilon^{n+2}\right)$. However, by (11) this influx will be $O\left(R^{-\frac{1}{3}}\right)$ $=O\left(\epsilon^{n+1}\right)$ unless the $O(1)$ circulation about the closed streamline is zero.

Thus, we are led again to (15) as the boundary condition to be applied on the closed portion of the critical streamline, provided the right side of (17) can be neglected. In proving this, we assume that the vorticity $\zeta^{(0)}$ is $O(1)$ as before, since the appropriate equation is still (13), but without the Ekman layer suction term. The thickness of layers across which $\dot{\zeta}^{(0)}$ may change abruptly is now $(2 \in R)^{-\frac{1}{2}}$, which is simply $(\operatorname{Re})^{-\frac{1}{2}}$, where $\mathrm{Re}=V H / \nu$ is the Reynolds number. Thus, the right side of (17) is $O\left(\epsilon^{\frac{1}{2}} R^{-\frac{1}{3}}\right)=O\left(\epsilon^{n+3 / 2}\right)$, which is small compared to $\epsilon^{n+1}$, and is therefore negligible.

In the inviscid limit (16), the equation to be solved is (13) with right side equal to zero, subject to the boundary condition at infinity, and to the free surface condition (15). The latter has a simple physical interpretation in terms of the pressure. If there is no motion within the Taylor column, the pressure $p$ must be constant around its edge. Thus, at the edge of the Taylor column we have

$$
\mathbf{q} \cdot \boldsymbol{\nabla} p=0
$$

which is satisfied automatically to lowest order in $\epsilon$, since to this order the edge of the Taylor column is a streamline $p^{(0)}=$ constant. The terms in (22) proportional to $\epsilon$ may be expressed in terms of $p^{(0)}$ by means of 


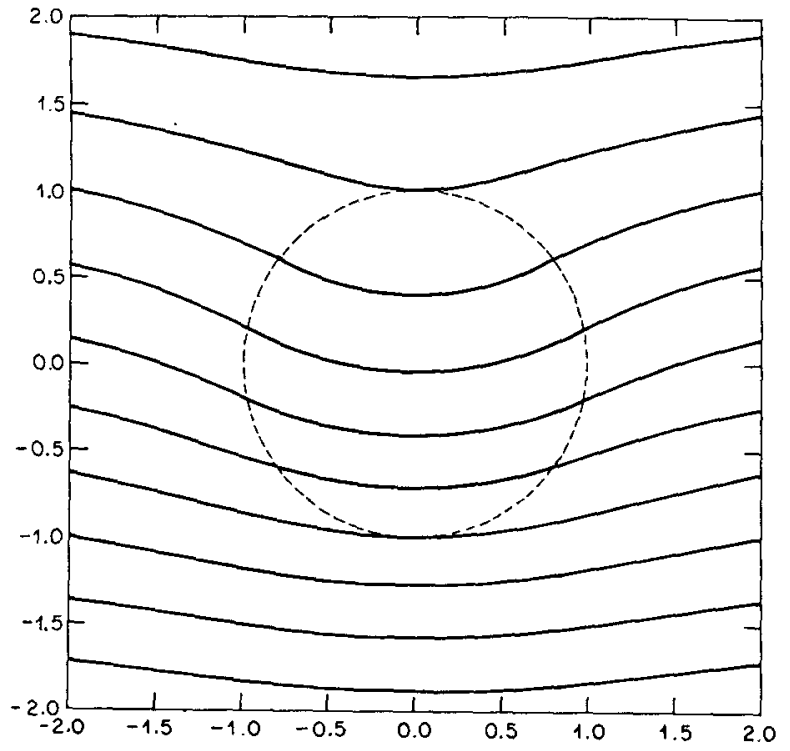

FIG. 1. Streamlines over a flat, cylindrical obstacle of height $h_{0}=\epsilon$. The flow is from right to left if the rotation is counterclockwise, left to right if the rotation is clockwise, and the separation of streamlines at infinity is 0.4 .

the Bernoulli equation (7). Thus, we have

$$
\mathbf{q} \cdot \nabla p=-\boldsymbol{\epsilon} \mathbf{q}^{(0)} \cdot \nabla E^{(0)}=0, \quad\left[p^{(0)}=p_{c}{ }^{(0)}\right],
$$

to first order in $\epsilon$. Thus, $E^{(0)}$ must be constant along the closed portion of the critical streamline, and since it is zero at the stagnation point, we have

$$
E^{(0)}=\frac{1}{2}\left|\nabla p^{(0)}\right|^{2}=0, \quad\left[p^{(0)}=p_{c}^{(0)}\right],
$$

which is the same as (15).

\section{Two examples}

The differential equation in the inviscid limit is simplified considerably if the flow is uniform at infinity. Eq. (13) with right side equal to zero implies that the quantity $\left[\zeta^{(0)}+h / \epsilon\right]$ is constant along streamlines, and is therefore zero everywhere outside the critical streamline. Thus, we have

$$
\zeta^{(0)}+h / \epsilon=\nabla^{2} \psi+h / \epsilon=0,
$$

where the lowest order pressure $p^{(0)}$ has been replaced by the symbol $\psi(x, y)$. Henceforth, we shall measure horizontal distances in uints of $L$, a characteristic length which may be different from the depth $H$. The stream function is measured in units of $L V$, and the height of the obstacle is measured in units of $H$. Then (25) is unchanged provided we use the Rossby number $\epsilon=V / 2 \Omega L$ based on the horizontal scale. We let the velocity at infinity be unity in nondimensional notation, directed along the negative $x$ axis. Then (25) must be solved subject to the boundary conditions

$$
\begin{gathered}
\psi_{y} \rightarrow 1, \quad \psi_{x} \rightarrow 0 \quad(r \rightarrow \infty), \\
\nabla \psi=0 \quad(\psi=0, \text { closed portion }) .
\end{gathered}
$$

Here we have introduced circular coordinates $r$ $=\left(x^{2}+y^{2}\right)^{\frac{1}{2}}, \Theta=\tan ^{-1} y / x$, and have arbitrarily set $\psi=0$ on the critical streamline. Note that both $\psi$ and its normal derivative must vanish on the closed curve at the edge of the Taylor column.

\section{a. Flat, cylindrical obstacle}

We set $h(r)=h_{0}=$ constant for $r<1$, and $h(r)=0$ for $r>1$. This example is somewhat artificial, because of the discontinuity in depth at $r=1$. However, a formal solution of (25)-(26) exists, as first pointed out by Venezian (private communication), and so the example is a useful one. When the height of the obstacle $h_{0}$ is less than $2 \epsilon$, the streamlines are distorted in the vicinity of the obstacle, but they do not cross (Fig. 1). In this case the solution is

$$
\psi= \begin{cases}-\frac{h_{0}}{2 \epsilon} \ln r+r \sin \Theta-1 & (r>1), \\ -\frac{h_{0}}{4 \epsilon}\left(r^{2}-1\right)+r \sin \Theta-1 & (r<1) .\end{cases}
$$

The boundary condition (26a) is sufficient to determine the flow everywhere in the $x-y$ plane. There is a clockwise circulation $\pi h_{0} / \epsilon$ around the body, and an associated force on the body $\pi h_{0}$ in the negative $y$ direction. (In the present notation, this is exactly the Coriolis force which would act on an equal volume of fluid flowing in the positive $x$ direction with the free stream velocity.)

When the height $h_{0}>2 \epsilon$, the solution (27) gives closed streamlines, but the condition (26b) is not satisfied on the critical streamline, and there is flow within the Taylor column. Eq. (27) is the only solution of (25) and (26a) which is regular everywhere in the $x-y$ plane, and is the solution usually offered in inviscid theories. However, (25) is not necessarily valid on closed streamlines, since these do not extend to $r \rightarrow \infty$; as we have shown, this fact is properly taken into account by applying (26b) on the closed portion of the critical streamline.

The correct solution for $h_{0}>2 \epsilon$ is

$$
\psi= \begin{cases}-\frac{h_{0}}{2 \epsilon} \ln r+\frac{K}{2 \pi} \ln \frac{r^{\prime}}{r_{c}}+r \sin \Theta-1 & (r>1), \\ -\frac{h_{0}}{4 \epsilon}\left(r^{2}-1\right)+\frac{K}{2 \pi} \ln \frac{r^{\prime}}{r_{c}}+r \sin \Theta-1 . & \left(r<1, r^{\prime}>r_{c}\right) .\end{cases}
$$

The Taylor column is a circle centered at $(x=0$, $\left.y=2 \epsilon / h_{0}\right)$, circumscribed within the obstacle and tangent to it at $(x=0, y=1)$. The radius of the Taylor column is $r_{c}=1-2 \epsilon / h_{0}$, and $r^{\prime}$ is the distance from its center to the point $(x=r \cos \theta, y=r \sin \Theta)$. Finally, the 
constant $K=\pi h_{0} r_{c}^{2} / \epsilon$ is the additional circulation necessary to satisfy (26b). The net clockwise circulation about the obstacle is $4 \pi\left(1-\epsilon / h_{0}\right)$, corresponding to a force $4 \pi \epsilon\left(1-\epsilon / h_{0}\right)$ acting in the negative $y$ direction. Examples of this solution are plotted in Figs. 2-3, for various values of $h_{0} / \epsilon$. For a high obstacle $\left(h_{0} / \epsilon \gg>1\right)$, the Taylor column coincides with the obstacle itself, and the force on the obstacle is $4 \pi \epsilon$ in the negative $y$ direction. (This is $4 \epsilon$ times the Coriolis force on a volume of fluid equivalent to the obstacle plus Taylor column.)

Recently, T. Maxworthy (private communication) has observed Taylor columns in the laboratory for the case $1 \gg \epsilon \gg R^{-\frac{1}{2}}$. He finds that stagnation first occurs above a lens-shaped body whose height relative to the container depth is roughly 3-4 times the Rossby number. Taking $h / \epsilon=C\left(1-r^{2}\right)$, we find from (25) and (26a), that stagnation is expected to occur for $C \geqq 3(3 / 2)^{\frac{1}{2}}$ $\approx 3.68$, in excellent agreement with observation. Maxworthy also finds that the sense of the asymmetry is the same as in Figs. 1-3 of this paper, with the circulation in a direction opposite the rotation, and the stagnant region of the right-looking downstream, for counterclockwise rotation. ${ }^{2}$

\section{b. General symmetric obstacle}

Exact solutions of (25)-(26) have only been obtained for the flat cylinder. However, it is possible to obtain approximate solutions valid for high obstacles (relative to $\epsilon$ ) which terminate abruptly at $r=1$. Consider the case $h(r)=0$ for $r>1$, and $h(r)=h_{0}{ }^{\prime}(1-r)+h_{0}{ }^{\prime \prime}(1-r)^{2} /$ $2+\cdots$, for $r<1$, where the slope $h_{0}{ }^{\prime}$ is large compared to $\epsilon$. Because the slope is large, we assume that the boundary of the Taylor column almost coincides with the edge

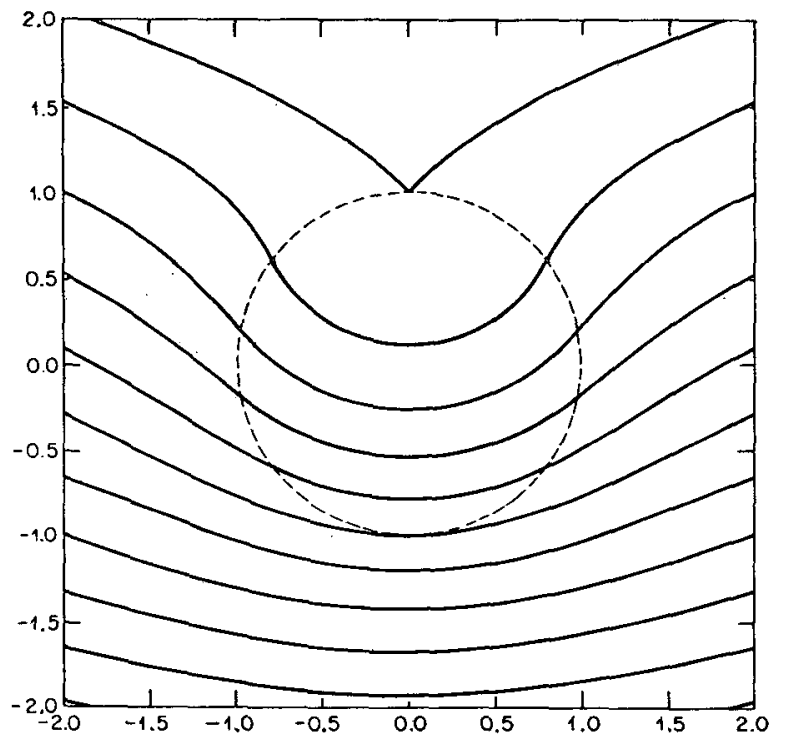

FIG. 2. Same as Fig. 1 except that $h_{0}=2 \epsilon$.

${ }^{2}$ Note added in proof.

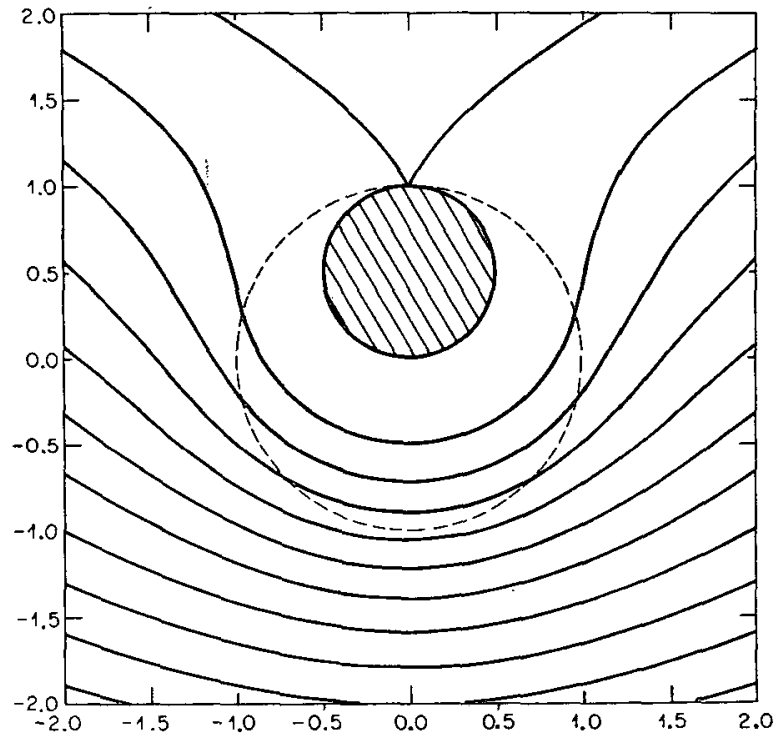

Frg. 3. Same as Fig. 1 except that $h_{0}=4 \epsilon$.

of the obstacle at $r=1$. Thus, an approximate solution is

$$
\begin{gathered}
\psi=\frac{C}{2 \pi} \ln r+\left(r-\frac{1}{r}\right) \sin \Theta \quad(r>1), \\
\psi=-\frac{h_{0}^{\prime}}{6 \epsilon}(1-r)^{3}+\frac{C}{2 \pi} \ln r+\left(r-\frac{1}{r}\right) \sin \Theta . \\
(0<1-r \ll 1),
\end{gathered}
$$

where $C$ is the circulation around the obstacle, to be determined. Note that $\psi$ and its normal derivative are continuous at $r=1$, and that the critical streamline $\psi=0$ coincides with the circle $r=1$.

However, (26b) is not satisfied exactly at $r=1$. In fact, we may use (26b) to determine the position of the Taylor column, and ultimately the circulation $C$. First note that no portion of the Taylor column may extend to $r>1$. In this region, $\psi$ satisfies LaPlace's equation, and the function and its normal derivative cannot be equal to zero on a finite curve. Therefore, from (29a) we have

$$
-2 \leqq C / 2 \pi \leqq 2 .
$$

We now use (29b) to derive an approximate expression for the curve $r_{c}(\Theta)$ on which (26b) is satisfied. Making use of the fact that $h_{0}{ }^{\prime} / \epsilon$ is large, we find

$$
\left(r_{c}-1\right)^{2}=-\frac{2 \epsilon}{h_{0}^{\prime}}\left[\frac{C}{2 \pi}+2 \sin \Theta\right] .
$$

But if $h_{0}^{\prime}>0$, (30) and (31) are consistent only when

$$
C=-4 \pi, \quad r_{c}(\Theta)=1-\left[\frac{4 \epsilon}{h_{0}{ }^{\prime}}(1-\sin \Theta]^{\frac{1}{2}} .\right.
$$


The solution for $r>1$ is the same as that obtained for the high flat cylinder $\left(h_{0} \gg \epsilon\right)$. The clockwise circulation is $4 \pi$, and the force on the obstacle is $4 \pi \epsilon$ in the negative $y$ direction.

It is convenient at this point to discuss the forces on obstacles. In the previous examples, where the flow is in the negative $x$ direction, the force on a stationary obstacle is in the negative $y$ direction. To interpret this physically, it is simpler to let the fluid be stationary at $r \rightarrow \infty$, and have the body moving in the positive $x$ direction. Derivatives of $\psi$ now give the velocity of the fluid relative to that of the body, and the pressure $p^{(0)}$ is now $\psi-y$. The force on the obstacle computed from the stream function $\psi$, as in the examples, is now the net force, including forces exerted by the fluid and the Coriolis force due to the motion of the body. For the flat cylinder with $h_{0}<2 \epsilon$ (no Taylor column), this net force is simply the Coriolis force $\pi h_{0}$ in the negative $y$ direction; the fluid exerts no force in this case. For $h_{0}>2 \epsilon$, the net force is less than the Coriolis force which would act on a volume of fluid equal to the body plus Taylor column. For $h_{0} \gg \epsilon$, the net force is $\mathrm{O}(\epsilon)$ times the Coriolis force; the fluid almost balances the Coriolis force due to the motion of the obstacle. This behavior was observed by Taylor (1923) in his experiments on towed spheres and cylinders.

\section{Geophysical applications}

Phillips (1963) gives equations appropriate to a stratified, inviscid ideal gas on a rotating sphere. These equations are based on an expansion in the Rossby number similar to that presented in this paper. The motion is still horizontal and nondivergent, to lowest order in the Rossby number, but now the lowest order stream function depends on the vertical coordinate. This simply reflects the fact that the Taylor-Proudman theorem (10) does not hold for a stratified fluid, and so Taylor columns will not form when stratification is important. We now describe the relevant nondimensional parameters which determine the importance of stratification, and of the spherical geometry.

We let $x$ and $y$ be horizontal coordinates eastward and poleward, respectively, scaled by a length $L$. The equations then hold over a limited portion of the sphere centered at latitude $\Phi_{0}$, for which the ratio $L / a<1$, where $a$ is the radius of the sphere. The Rossby number is now based on the vertical component of the rotation vector at latitude $\Phi_{0}, \epsilon=V /\left|2 \Omega L \sin \Phi_{0}\right|$. The height of the obstacle $h$ is measured relative to the scale height $H=R T / g$, evaluated at the surface, where $T$ is the temperature, $g$ the acceleration of gravity, and $R$ the gas constant of the atmosphere. Finally, we let $z$ be the vertical coordinate, normal to the surface of the sphere, and $c_{p}$ the specific heat of the gas at constant pressure. Then the effects of stratification and spherical geometry ( $\beta$ effect) depend on the magnitude of the parameters $B$ and $b$, where

$$
B=\frac{g H^{2}}{\left(2 \Omega L \sin \Phi_{0}\right)^{2}} \frac{1}{T}\left(\frac{d T}{d z}+\frac{g}{c_{p}}\right), \quad b=L /(a \epsilon) .
$$

In deriving the basic equations, Phillips assumes that $B$ and $b$ are $O(1)$, with $H / L, L / a$, and $\epsilon$ all small. To this approximation, the coordinates $x, y, z$ define a Cartesian system; the only effect of the spherical geometry is that the vorticity $\zeta$ is replaced by $\zeta+b y$, where $y$ is the poleward coordinate measured from the mean latitude $\Phi_{0}$. This is known as the $\beta$-plane approximation.

The effects of stratification and spherical geometry are small when $B$ and $b$ are small, but Phillips does not present a scheme for proceeding to the limit as $B$ and $b$ tend to zero. In what follows, we shall simply assume that stratification and spherical geometry can be neglected when $B$ and $b$ are small. This assumption should be verified; the case $B \rightarrow 0$ is especially troublesome because of difficulties with the boundary condition at $z \rightarrow \infty$. It is possible that the effects of stratification are never negligible as $B \rightarrow 0$, at sufficient heights in the atmosphere.

The effects of vertical shear due to horizontal temperature differences (baroclinicity) can be estimated by the relation

$$
\Delta V / V \approx(B / \epsilon)\left(\Delta T_{h} / \Delta T_{v}\right),
$$

where $\Delta V$ is a typical difference in horizontal velocity over one scale height $H$, and $\Delta T_{h}$ and $\Delta T_{v}$ are typical horizontal and vertical temperature differences over distances $L$ and $H$, respectively. We shall assume that baroclinicity is unimportant when $B / \epsilon<1$, since $\Delta T_{h} / \Delta T_{v}$ is typically $\mathrm{O}(L / a)$, a small quantity.

We now discuss the magnitude of the constants $\epsilon, B$ and $b$ for the atmospheres of the earth and Jupiter. For the earth, taking $V=15 \mathrm{~m} \mathrm{sec}^{-1}, L=10^{3} \mathrm{~km}, H=8 \mathrm{~km}$, $\Phi_{0}=30^{\circ}$ and $d T / d z=-6.5 \mathrm{C} \mathrm{km}^{-1}$, we obtain

$$
\epsilon \approx 0.2, \quad B \approx 1.5, \quad b \approx 0.8 \text {. }
$$

Thus, the expansion in $\epsilon$ is only a rough approximation and the effects of stratification, baroclinicity and spherical geometry ( $\beta$ effect) are all important. Because of stratification, the flow will not be two-dimensional, and the Taylor-Proudman theorem (10) will not hold. This fact probably explains why Taylor columns are not observed on the earth.

The main difference between the earth's atmosphere and Jupiter's is the immense horizontal scale of phenomena on Jupiter. The Great Red Spot covers some $10^{\circ}$ of latitude and $30^{\circ}$ of longitude, corresponding to $L \approx 10^{4} \mathrm{~km}$. And taking $V=50 \mathrm{~m} \mathrm{sec}^{-1}$ (Reese and Smith, 1968), $H=8 \mathrm{~km}$ (Öpik, 1962), $d T / d z=0$ (extreme stratification), and $c_{p} / R=3.5$ (diatomic gas), we obtain

$$
\epsilon \approx 0.04, \quad B \approx 0.03, \quad b \approx 4 .
$$


These are conservative estimates; if the velocity is lower (Hide, 1963), and the lapse rate $d T / d z$ is more nearly adiabatic, $\epsilon$ and $B$ will be smaller, and $b$ will be larger. Thus, stratification and baroclinicity are not likely to be important for large scale phenomena such as the Great Red Spot. We therefore assume that the stream function $\psi(x, y)$ is independent of the vertical coordinate, and obeys the equation

$$
\mathbf{q} \cdot \mathbf{V}(\zeta+b y+h / \epsilon)=0,
$$

where $u=-\psi_{y}, v=\psi_{x}$, and $\zeta=\nabla^{2} \psi$, as before. With these definitions, (37) is valid in both the Northern and Southern Hemispheres, provided $x$ is always the eastward (prograde) coordinate, and $y$ is always the poleward coordinate. Note that (37) is identical to (13) in the inviscid limit, with an additional term $b y$ due to the spherical geometry.

We are assuming that there is a rigid surface below Jupiter's atmosphere, the equation of which is $z=h(x, y)$. We also assume that this surface exerts a small frictional drag on the overlying fluid, whence the boundary condition at the edge of the Taylor column (15). In short, we are investigating the implications of Hide's hypothesis that the Spot is a Taylor column formed as a result of interaction between the atmosphere and the surface of Jupiter. Problems concerning the nature and motion of this surface have been discussed by Hide, and will not be mentioned here.

We let the stream function at infinity be $U y$, corresponding to uniform flow in the negative $x$ direction (retrograde), if $U$ is positive. Then (37) reduces to

$$
\nabla^{2} \psi-b(\psi-U y) / U+h / \epsilon=0
$$

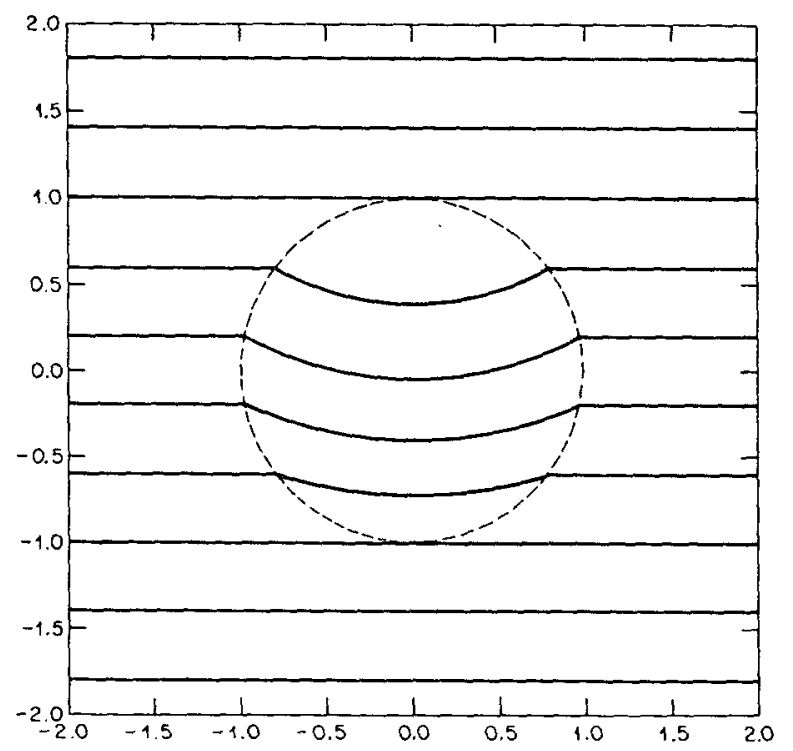

FIG. 4. Streamlines on a $\beta$ plane for retrograde flow over an obstacle of height $h(r)=(L / 4 a)\left(1-r^{2}\right)$. The flow is from east to west (right to left in the Northern Hemisphere, left to right in the Southern Hemisphere), and the separation of streamlines at infinity is 0.4. A smooth transition at $r=1$ takes place in an inertial boundary layer of thickness $(\epsilon a / L)^{1}$.

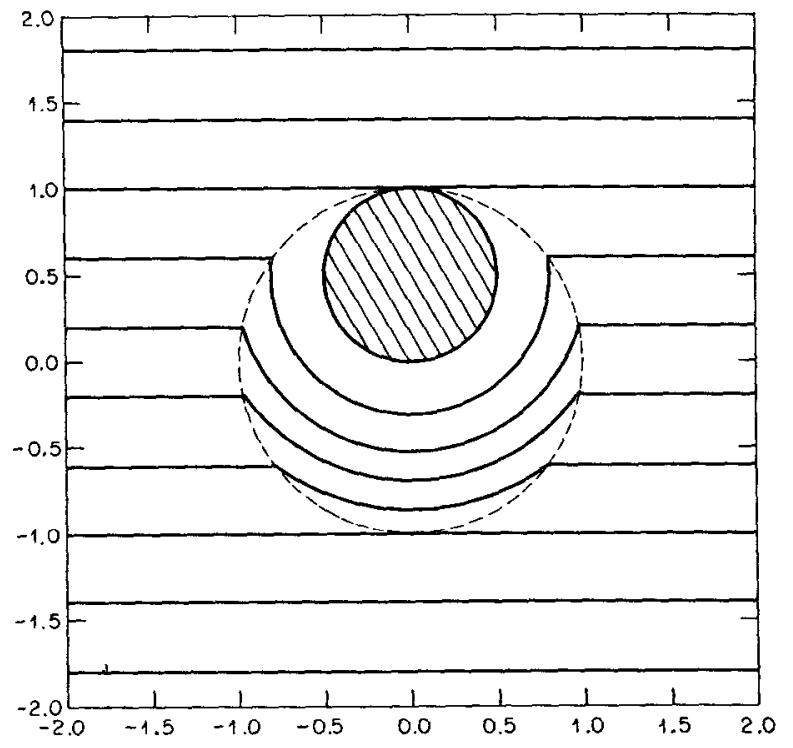

FIG. 5. Same as Fig. 4 except for $h(r)=(L / a)\left(1-r^{2}\right)$. Inertial boundary layers form at $r=1$ and at the edge of the stagnant region.

which must be solved subject to the boundary conditions

$$
\begin{gathered}
\psi_{y} \rightarrow U, \quad \psi_{x} \rightarrow 0 \quad(r \rightarrow \infty), \\
\nabla \psi=0 \quad(\psi=0, \text { closed portion }) .
\end{gathered}
$$

Eq. (38), like (25), is linear in $\psi$ with constant coefficients, but the free surface boundary condition $(39 \mathrm{~b})$, like (26b), introduces a critical nonlinearity into the problem. The only simple case which can be analyzed occurs when $b / U$ is large and positive (retrograde flow). An approximate solution of (38) and (39a) is then

$$
\psi_{1}=U y+h U /(b \boldsymbol{\epsilon}) .
$$

There is no circulation about the object at $r \rightarrow \infty$, and streamlines $\psi_{1}=$ constant are deflected toward the equator by an object with $h>0$ (Figs. 4-5). For retrograde flow, the asymmetry to the left and right of the obstacle is the same as for solutions of (25). Closed streamlines will appear for $h=O(\epsilon b)=O(L / a)$, where $h$ is the height of the obstacle relative to the scale height $H=R T / g$. For the Great Red Spot, this critical height is about $2 \mathrm{~km}$.

However, the solution (40) does not satisfy (39b) on the closed portion of the critical streamline. In fact, an inertial boundary layer of thickness $(U / b)^{\frac{1}{2}}$ develops in the vicinity of this streamline. Within the inertial boundary layer, complementary solutions appear which satisfy the equation

$$
\nabla^{2} \psi_{2}-(b / U) \psi_{2}=0
$$

and which vanish exponentially at the outer edge of the layer. It is not difficult to show that the location of the streamlines is adequately represented by (40), although 
the velocity components obtained from (40) are only correct outside the inertial boundary layer.

If the flow is prograde relative to the obstacle $(U<0)$, solutions of (41) are no longer confined to boundary layers, but exhibit wavelike behavior. A similar situation exists at the western boundaries of ocean basins where the flow in the open ocean is to the east (e.g., Carrier and Robinson, 1962). Such regions are not well understood, but it appears that simple relations of the form (40) are not valid everywhere in the interior of the fluid. Thus, when $b / U$ is large and the flow is prograde relative to the obstacle, steady inviscid Taylor columns may not exist, or they may be very different from the examples given here.

The flow around Jupiter's Great Red Spot bears some resemblance to the solutions (40) for retrograde flow (Fig. 5). In the first place, the parameter $b / U$ is large, as implied in (36). Second, the streamlines are clearly deflected towards the equator, as implied by the existence of the Red Spot Hollow on the equatorward side of the Spot. Third, Reese and Smith (1968) report repeated observations of counterclockwise circulation around the edge of the Spot, which is only consistent with the present theory if the flow is retrograde. On the other hand, the observed east-west velocity at the latitude of the Spot is more complicated. Equatorward of the Spot, in the South Equatorial Belt, extreme retrograde motion is observed with rotation periods greater than $9^{h} 58^{m}$ (Peek, 1958; Reese and Smith, 1968). Poleward of the Spot, in the South Temperate Belt, extreme prograde motion is observed with rotation periods about $9^{h} 53^{m}$. For comparison, the mean rotation period of the Spot during the last hundred years is $9^{h} 55^{m} 38^{s}$.

The fact that there is prograde flow at the poleward edge of the Spot means that simple solutions of the form (40) are not valid over the entire region. Further theoretical work is needed to understand the effects of nonuniform, prograde flow at infinity, as well as to verify that stratification can be neglected for small values of $B$. The motion of a floating body on a $\beta$ plane should also be investigated. In this regard, the fact that the Spot lies in a zone of extreme negative relàtive vorticity (taking the planetary vorticity to be positive) may prove significant.

Acknowledgments. The author wishes to thank $\mathrm{Dr}$. Giulio Venezian for his many helpful suggestions, and for the interest he has shown in this work. Financial support was provided partly by the National Aeronautics and Space Administration under Grant NGL 05-002-003.

\section{REFERENCES}

Benney, D. J., 1965: The flow induced by a disk oscillating about a state of steady rotation. Quart. J. Mech. Appl. Math., 18, 333-34.5.

Carrier, G. F., and A. R. Robinson, 1962: On the theory of the wind-driven ocean circulation. J. Fluid Mech., 12, 49-80.

Greenspan, H. P., and L. N. Howard, 1963: On a time-dependent motion of a rotating fiuid. $J$. Fluid Mech., 17, 385-404.

- , and $S$. Weinbaum, 1965: On non-linear spin-up of a rotating fluid, J. Math. Phys., 44, 66-85.

Hide, R., 1961 : Origin of Jupiter's Great Red Spot. Nature, 190, 895-896.

- 1963: On the hydrodynamics of Jupiter's atmosphere. La Physique des Planetes, Belgium, Institut d'Astrophysique, Cointe-Sclessin, 481-505.

columns." Icarus, 5, 279-290.

- - - and M. J. Lighthill, 1968: On slow transverse flow past obstacles in a rapidly rotating fluid. J. Fluid Mech., 32, 251272 .

Jacobs, S. J., 1964: The Taylor column problem. J. Fluid Mech, 20, 581-591.

Öpik, E. J., 1962 : Jupiter: Chemical composition, structure, and origin of a giant planet. Icarus, 1, 200-257.

Peek, B. M., 1958: The Planet Jupiter. London, Faber and Faber, $283 \mathrm{pp}$.

Phillips, N. A., 1963: Geostrophic motion. Rev. Geopllys., 1, 123176.

Proudman, J., 1916: On the motion of solids in a liquid possessing vorticity. Proc. Roy. Soc. (London), A92, 408-424.

Reese, E. J., and B. A. Smith, 1968: Evidence of vorticity in the Great Red Spot of Jupiter. Icarus, 9, 474-486.

Stewartson, K., 1953: On the slow motion of an ellipsoid in a rotating fluid. Quart. J. Mech. Appl. Math., 6, 141-162.

Taylor, G. I., 1917: Motion of solids in fluids when the flow is not irrotational. Proc. Roy. Soc. (London), A93, 99-113.

_ 1923 : Experiments on the motion of solid bodies in rotating fluids. Proc. Roy. Soc. (London), A104, 213-218. 